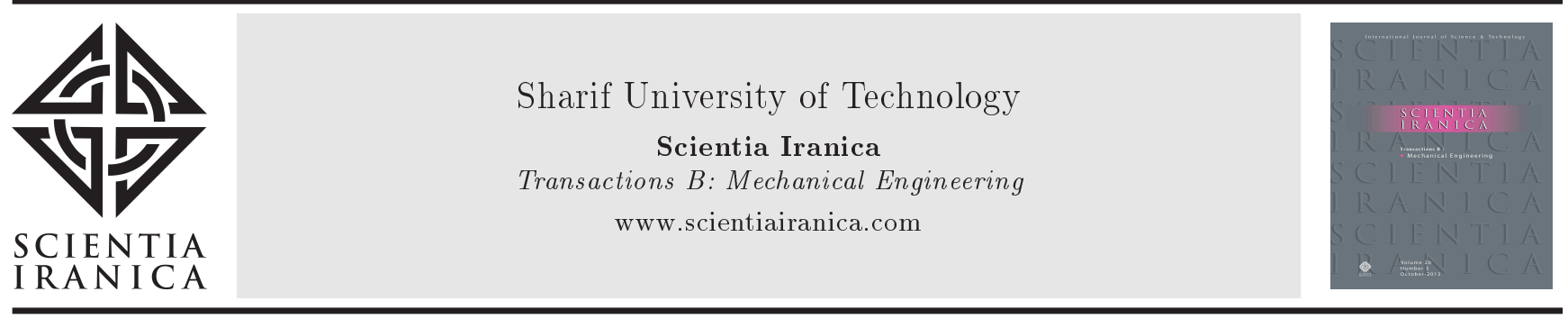

\title{
High-frequency vibrations of quartz crystals subject to initial thermo-mechanical bias
}

\author{
M.M. Mohammadi, M. Hamedi* and H. Daneshpajooh \\ School of Mechanical Engineering, College of Engineering, University of Tehran, Tehran, P.O. Box 11155/4563, Iran. \\ Received 29 October 2015; received in revised form 7 April 2016; accepted 2 May 2016
}

\section{KEYWORDS}

Quartz;

Force-frequency effect;

Thermal bias;

Mathematical

modelling.

\begin{abstract}
This paper is concerned with analyzing the resonance frequency change of a quartz resonator under initial thermal and mechanical biases. The change in the resonance frequency of the resonator subjected to a pair of diametrical forces is called the forcefrequency effect and is quantified by force-frequency coefficient. The experimental data suggest that this coefficient may change due to homogenous thermal biases on the quartz crystal. In this article, we investigate the effect of initial homogenous thermal strains on the force-frequency effect. We derive an explicit formula that predicts the resonance frequency shift of the thickness-shear mode of AT-cut quartz crystals. The mathematical model is validated on circular AT-cut quartz crystals at $78^{\circ} \mathrm{C}$ using the experimental results. The model leads to a better understanding of quartz crystal behavior which increases accuracy of pressure sensors in applications such as down-hole pressure measurement in the oil and gas industry.

(C) 2017 Sharif University of Technology. All rights reserved.
\end{abstract}

\section{Introduction}

Quartz crystal has important properties like the existence of zero temperature cuts, low intrinsic acoustic loss, and low cost [1]. Many quartz crystal resonators, as shown in Figure 1(a), operate with thickness-shear (T-Sh) vibration mode $[2,3]$. When a quartz resonator is subjected to diametrical forces, its resonance frequency changes. This change in the resonance frequency is referred to as the force-frequency effect [4]. Currently, many sensors work on the principle of forcefrequency effect [5-8]. Figure 1(b) shows a quartz resonator pressure sensor which is used for measuring down-hole oil pressure through the application of forcefrequency effect.

In this type of sensor, the oil pressure acts on the external surface of the cylindrical body and is transmit-

* Corresponding author.

E-mail addresses: mmmohamamdi@ut.ac.ir (M.M.

Mohammadi); mhamedi@ut.ac.ir (M. Hamedi);

hossein.daneshpajooh@gmail.com (H. Daneshpajooh) ted to the circular quartz resonator, hence changing the resonance frequency of the resonator. The resonance frequency shift of the resonator has a linear dependency on the applied pressure and is measured as the output of the sensor [7], which enables the sensors to be used for measuring pressure. The change of temperature affects the frequency shift of the sensor at various temperatures. Accordingly, understanding the effect of temperature on the force-frequency phenomena is an important aspect of designing quartz crystal sensors that can accurately measure the pressure at various temperatures.

Rajitsky defined the force-frequency coefficient in 1965 and measured the coefficient for single rotated quartz crystals [9]. Dauwalter described the effect of temperature change on the force-frequency coefficient [10]. He demonstrated that the temperature dependence of the force-frequency coefficient depends on the azimuth angle, $\psi$, between the $X$ crystallographic axis and the direction of the applied force. The theoretical aspect of the problem belongs to the general theory of incremental elastic deformations 


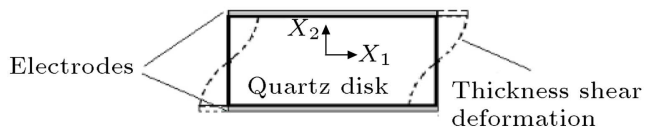

(a)

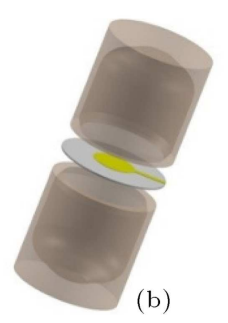

Figure 1. (a) Thickness-shear deformation in a quartz crystal resonator. (b) A down-hole sensor working with quartz crystal resonator.

superposed on initial finite deformations [11]. The initial deformation is produced by applying diametric forces and also depends on the change in temperature of the medium. To the best of our knowledge, researchers have considered effects of force-frequency and temperature frequency separately. However, thermal bias affects the force-frequency coefficient that has to be studied explicitly, and this can merit further research.

The general theory of incremental elastic deformations superposed on initial finite deformations, which can be of both thermal and mechanical types, has been studied [12-14]. One of the main methods for analyzing the effects of force-frequency and temperature frequency was proposed by Lee et al. [11]. Lee developed the basic equations of the theory in the Lagrangian form to analyze effect of the force-frequency at room temperature. In the nonlinear stress-strain relations, the Third-Order Elastic coefficients (TOEs) were included. In his study, an explicit formula was obtained for predicting changes in the thickness-shear frequencies by applying diametric forces. In 1986, in another work, a three-dimensional equation of motion for small vibration superposed on finite homogenous thermal deformation was derived by Lee and Yong [15] who considered the material properties of quartz as being temperature-dependent. By obtaining the relation of resonance frequency to the initial temperature of the plate, Lee succeeded in calculating six values for the effective third temperature derivatives of elastic stiffness $(\tilde{C})$.

In this paper, we focus on the effect of initial homogenous thermal strains on the force-frequency coefficient of quartz disks. We extracted two-dimensional equations for high-frequency vibrations of crystal plates under initial mechanical stresses and homogenous thermal strains by Mindlin procedure. These equations were applied for thickness-shear vibrations of rotated Y-Cut quartz crystals. We derived an analytical equation for calculating the change of fundamental thickness-shear frequencies in terms of initial thermal and mechanical strains and the second and third order elastic constants for rotated Y-Cut quartz crystals. We checked the developed equations with published experimental results on circular AT-cut quartz crystals.

The contributions of this paper can be outlined as follows:

- Two-dimensional equation of motion and traction boundary condition for crystal plates with arbitrary crystallographic symmetry, which are under mechanical and homogenous thermal biases, are developed. Also, the $n$ th-order components of the second Piola-Kirchhoff stress tensor are obtained;

- The ensuing equations are employed to derive a formula for calculating the resonance frequency shift of rotated Y-Cut quartz crystals subjected to initial mechanical and homogenous thermal biasing fields;

- The force-frequency coefficients of a circular AT-Cut quartz crystal at temperature $78^{\circ} \mathrm{C}$ are calculated, and some suggestions are made for better design of AT-Cut resonators and sensors.

\section{Three-dimensional motion equations}

As mentioned in the introduction, the analysis of the effect of the force-frequency on piezoelectric resonators belongs to the general theory of incremental elastic deformations superimposed on initial finite deformations. The basic equations of this theory are developed in the Lagrangian form. In nonlinear stress-strain relations, TOEs are included. The material constants in the governing equations are temperature-dependent. Moreover, homogenous thermal and mechanical strains must be taken into account. By employing power series expansions of displacements and body forces in variational principle, two dimensional motion equations for the quartz resonator are derived.

Upon the Lagrangian description, the crystal is considered to be in three states, which are natural, initial, and final states. At the natural state, the crystal has a constant uniform temperature, $T_{0}$, and experiences no displacement, strain, or stress. At the initial state, the body is subjected to thermal and mechanical deformations due to temperature change and the application of diametric forces. At the final state, the body is subjected to a small-amplitude vibration in addition to the thermal and mechanical deformations being imposed on the initial state.

Figure 2 shows position vectors $x_{i}, y_{i}$, and $z_{i}$ of material points at the natural, initial, and final states, respectively.

In Eq. (1), the capital letters denote the initial state, the barred capital letters represent the final state, and the lowercase letters are used for the incremental quantity with more details in [11]. The incremental quantities can be obtained by subtracting the initial 


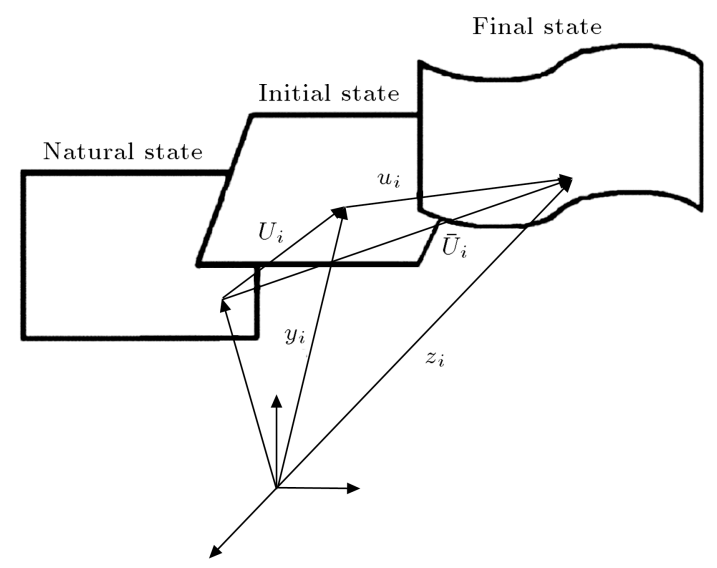

Figure 2. Position vectors for material points at the natural, initial, and final states.

state from the final state $[16,17]$. The final, initial, and incremental strains are given by the gradient equations:

$$
\begin{aligned}
\bar{E}_{i j} & =\frac{1}{2}\left(\bar{U}_{i, j}+\bar{U}_{j, i}+\bar{U}_{k, i} \bar{U}_{k, j}\right), \\
E_{i j} & =\frac{1}{2}\left(U_{i, j}+U_{j, i}+U_{k, i} U_{k, j}\right), \\
\eta_{i j} & =\frac{1}{2}\left(u_{i, j}+u_{j, i}+U_{k, i} u_{k, j}+u_{k, i} U_{k, j}\right),
\end{aligned}
$$

where ()$_{, i}=\partial() / \partial x_{i}$. Also, $\bar{U}_{i, j}, U_{i, j}$, and $u_{i, j}$ are displacement gradients of the final, initial states, and incremental deformations, respectively. The kinetic and strain energy densities at the final state can be expressed as:

$$
\begin{aligned}
& K=\frac{1}{2} \rho \dot{\bar{U}}_{i} \dot{\bar{U}}_{i}, \\
& V=\frac{1}{2} C_{i j k l}^{\theta} \bar{E}_{i j} \bar{E}_{k l}+\frac{1}{6} C_{i j k l m n}^{\theta} \bar{E}_{i j} \bar{E}_{k l} \bar{E}_{m n}-\lambda_{i j}^{\theta} \bar{E}_{i j} .
\end{aligned}
$$

In the above equations, $\lambda_{i j}^{\theta}$ is the stress coefficient of temperature [18]; $C_{i j k l}^{\theta}$ as well as $C_{i j k l m n}^{\theta}$ are the second- and third-order elastic coefficients of quartz at temperature $\theta$. Applying the variational principle to the kinetic and strain energy densities, the threedimensional equation of motion and also the boundary condition for the final state can be obtained as $[11,18]$ :

$$
\begin{gathered}
\int_{t_{0}}^{t_{1}} d t \int_{v}\left[\left(\bar{T}_{j i}+\bar{T}_{j k} \bar{U}_{i, k}\right)_{, j}+\rho \bar{B}_{i}-\rho \ddot{\bar{U}}_{i}\right] \delta \bar{U}_{i} d v \\
+\int_{t_{0}}^{t_{1}} d t \int_{A}\left[\bar{Q}_{i}-n_{j}\left(\bar{T}_{j i}+\bar{T}_{j k}\right.\right. \\
\left.\left.\bar{U}_{i, k}\right)\right] \delta \bar{U}_{i} d A=0,
\end{gathered}
$$

where $\rho, \bar{E}_{i j}, \bar{T}_{j i}, \bar{U}_{i}, \bar{B}_{i}, \bar{Q}_{i}, v, A$, and $n_{j}$ are material density, the components of strain, the second Piola-Kirchhoff stress tensor, mechanical displacement, body force, surface traction, volume, surface area, and unit outward normal vector, respectively [11]. Due to very weak piezoelectricity of quartz, the coupling of mechanical field with electric field is neglected [11,15].

\section{Two-dimensional motion equations}

In this section, the two-dimensional equations for the plate are obtained by using power-series expansions of the displacements and body forces in the variational principle. Schematic view of an elastic plate of thickness, $2 b$, at its natural state, that is $x_{i}$ coordinates, can be seen in Figure 3. The body has the volume of $\tau$ and the middle plane area of $A$. The letter $C$ refers to the intersection of the cylindrical boundary of the plate with $A$ in the natural configuration. Accordingly, we have [19]:

$$
\begin{aligned}
& \left(\bar{U}_{i}, U_{i}, u_{i}\right)=\sum_{n} x_{2}^{n}\left(\bar{U}_{i}^{(n)}, U_{i}^{(n)}, u_{i}^{(n)}\right), \\
& \left(\bar{B}_{i}, B_{i}, b_{i}\right)=\sum_{n} x_{2}^{n}\left(\bar{B}_{i}^{(n)}, B_{i}^{(n)}, b_{i}^{(n)}\right) .
\end{aligned}
$$

In these equations, $\bar{U}_{i}^{(n)}, \bar{B}_{i}^{(n)}$ and $n=0,1,2, \ldots$ are the $n$ th-order components of displacements and body forces and are functions of $x_{1}, x_{3}$, and $t$. By substitution of the first relation of Eq. (4) into Eq. (1), we obtain:

$$
\left(\bar{E}_{i j}, E_{i j}, \eta_{i j}\right)=\sum_{n} x_{2}^{n}\left(\bar{E}_{i j}^{(n)}, E_{i j}^{(n)}, \eta_{i j}^{(n)}\right),
$$

where $\bar{E}_{i j}^{(n)}, E_{i j}^{(n)}$, and $\eta_{i j}^{(n)}$ are the $N$ th-order components of the final, initial, and incremental strains, respectively. For the strain components in Eq. (5), we have $[11,19,20]$ :

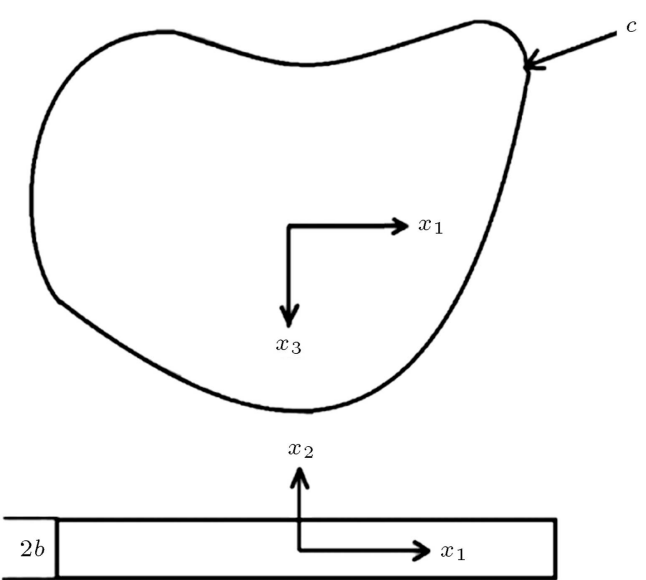

Figure 3. Two-dimensional quartz plate geometry. 


$$
\begin{aligned}
2 E_{i j}^{(n)}= & U_{j, i}^{(n)}+U_{i, j}^{(n)}+(n+1)\left(\delta_{2 i} U_{j}^{(n+1)}\right. \\
& \left.+\delta_{2 j} U_{i}^{(n+1)}\right)+\sum_{m}\left[U_{k, i}^{(m)} U_{k, j}^{(n-m)}\right. \\
& +m \delta_{2 i} U_{k}^{(m)} U_{k, j}^{(n-m+1)}+(n-m+1) \\
& \times \delta_{2 j} U_{k}^{(n-m+1)} U_{k, i}^{(m)}-m(n-m+2) \\
& \left.\delta_{2 i} \delta_{2 j} U_{k}^{(m)} U_{k}^{(n-m+2)}\right] \\
2 \eta_{i j}^{(n)}= & u_{j, i}^{(n)}+u_{i, j}^{(n)}+(n+1)\left(\delta_{2 i} u_{j}^{(n+1)}\right. \\
& \left.+\delta_{2 j} u_{i}^{(n+1)}\right)+\sum_{m}\left[U_{k, i}^{(m)} u_{k, j}^{(n-m)}\right. \\
& \left.\left.+u_{k}^{(m)} U_{k}^{(n-m+2)}\right)\right] \\
& +U_{k, j}^{(m)} u_{k, i}^{(n-m)}+m \delta_{2 i}\left(U_{k}^{(m)} u_{k, j}^{(n-m+1)}\right. \\
& \left.+\delta_{2 j}^{(m)} U_{k, j}^{(n-m+1)}\right)+(n-m+1) \\
& \left.U_{k}^{(n-m+1)} u_{k, i}^{(m)}+u_{k}^{(n-m+1)} U_{k, i}^{(m)}\right) \\
& \\
& \\
& \\
& \\
& \\
& \\
& \\
&
\end{aligned}
$$

where $\delta_{i j}$ is the Kronecker delta and $m=1,2,3, \ldots$.

The equation for $\bar{E}_{i j}^{(n)}$ is obtained by replacing the unbarred quantities of the first relation of Eq. (6) with barred quantities.

Two-dimensional kinetic and potential energy densities are defined as:

$$
\begin{aligned}
& \tilde{K}=\int_{-b}^{b} K d x_{2}, \\
& \tilde{V}=\int_{-b}^{b} V d x_{2} .
\end{aligned}
$$

By substituting Eq. (4) into the first relation of Eq. (2) and the resultant equation into the first relation of Eq. (7), we obtain:

$$
\tilde{K}=\frac{1}{2} \rho \sum_{m, n} a_{(m n)} \dot{\bar{U}}_{i}^{(m)} \dot{U}_{i}^{(n)}
$$

where:

$$
a_{(m n)}= \begin{cases}2 b^{m+n+1} /(m+n+1), & m+n=\text { even } \\ 0, & m+n=\text { odd }\end{cases}
$$

To obtain the strain energy density, the term for the stress coefficient of temperature in the second relation of Eq. (2) should be involved. An approximate relationship exists for the stress coefficient of temperature, which is $[15,21]$ :

$$
\lambda_{i j}^{\theta}=C_{i j k l}^{\theta} \alpha_{k l}^{\theta} \Delta T+\frac{1}{2} C_{i j k l m n}^{\theta} \alpha_{k l}^{\theta} \alpha_{m n}^{\theta}(\Delta T)^{2},
$$

where $\Delta T$ is the temperature change from the natural to the final states, and $\theta$ is the temperature at the final state, which is equal to the temperature at the initial state. The parameter $\alpha_{k l}^{\theta}$ is the thermal expansion coefficient at temperature $\theta$. By substituting Eq. (10) into the second relation of Eq. (2), we have:

$$
\begin{aligned}
V= & \frac{1}{2} C_{i j k l}^{\theta} \bar{E}_{i j}\left(\bar{E}_{k l}-2 \bar{E}_{k l}^{\prime}\right) \\
& +\frac{1}{6} C_{i j k l m n}^{\theta} \bar{E}_{i j}\left(\bar{E}_{k l} \bar{E}_{m n}-3 \bar{E}_{k l}^{\prime} \bar{E}_{m n}^{\prime}\right),
\end{aligned}
$$

where:

$$
\begin{aligned}
& {\overline{E^{\prime}}}_{k l}=\alpha_{k l}^{\theta} \Delta T, \\
& {\overline{E^{\prime}}}_{m n}^{\prime}=\alpha_{m n}^{\theta} \Delta T .
\end{aligned}
$$

By substituting Eq. (11) into the second relation of Eq. (7), we obtain:

$$
\begin{aligned}
\tilde{V}= & \frac{1}{2} \sum_{p, q} a_{p q} C_{i j k l}^{\theta} \bar{E}_{i j}^{(p)} \bar{E}_{k l}^{(q)} \\
& +\frac{1}{6} \sum_{p, q, r} a_{p q r} C_{i j k l m n}^{\theta} \bar{E}_{i j}^{(p)} \bar{E}_{k l}^{(q)} \bar{E}_{m n}^{(r)} \\
& -\sum_{p, q} a_{p q} C_{i j k l}^{\theta} \bar{E}_{i j}^{(p)} \bar{E}_{k l}^{\prime(q)} \\
& -\frac{1}{2} \sum_{p, q, r} a_{p q r} C_{i j k l m n}^{\theta} \bar{E}_{i j}^{(p)} \bar{E}_{k l}^{(q)} \bar{E}_{m n}^{(r)},
\end{aligned}
$$

where $a_{p q}$ is the same that as in Eq. (9), and $a_{p q r}$ is obtained by:

$$
\begin{aligned}
& a_{(p q r)}= \\
& \begin{cases}2 b^{p+q+r+1} /(p+q+r+1), & p+q+r=\text { even } \\
0, & p+q+r=\text { odd }\end{cases}
\end{aligned}
$$

The variational principle can be written in a form that is suitable for converting the three-dimensional equations to two-dimensional ones $[11,22]$ : 


$$
\begin{aligned}
\delta \int_{t_{0}}^{t_{1}} d t & \int_{A}(\tilde{K}-\tilde{V}) d A=\int_{t_{0}}^{t_{1}} d t\left\{\int _ { A } \left(\left[\bar{Q}_{i} \delta \bar{U}_{i}\right]_{X_{2}=b}\right.\right. \\
& \left.+\left[\bar{Q}_{i} \delta \bar{U}_{i}\right]_{X_{2}=-b}\right) d A+\int_{C} \int_{-b}^{b} \bar{Q}_{i} \delta \bar{U}_{i} d x_{2} d C \\
& \left.+\int_{A} \rho \bar{B}_{i} \delta \bar{U}_{i} d x_{2} d A\right\} .
\end{aligned}
$$

By applying Eq. (8), the variation of two-dimensional kinetic energy is obtained by:

$$
\int_{t_{0}}^{t_{1}} \delta \tilde{K} d t=-\rho \int_{t_{0}}^{t_{1}} \sum_{m, n} a_{(m n)} \ddot{\bar{U}}_{j}^{(m)} \delta \bar{U}_{j}^{(n)} d t .
$$

By virtue of Eq. (13), the variation of potential energy can be written as [23]:

$$
\delta \tilde{V}=\sum_{n} \frac{\partial \tilde{V}}{\partial \bar{E}_{i j}^{(n)}} \delta \bar{E}_{i j}^{(n)} .
$$

We define the $n$ th-order components of the stresses as $[11,19,23]$ :

$$
\left(\bar{T}_{i j}, T_{i j}, t_{i j}\right)=\int_{-b}^{b} x_{2}^{n}\left(\bar{T}_{i j}^{(n)}, T_{i j}^{(n)}, t_{i j}^{(n)}\right) d x_{2},
$$

which results in:

$$
\begin{aligned}
\bar{T}_{i j}^{(p)}= & \frac{\partial \tilde{V}}{\partial \bar{E}_{i j}^{(p)}}=\sum_{q} a_{p q} C_{i j k l}^{\theta}\left(\bar{E}_{k l}^{(q)}-\bar{E}_{k l}^{(q)}\right) \\
& +\frac{1}{2} \sum_{p, q, r} a_{p q r} C_{i j k l m n}^{\theta}\left(\bar{E}_{k l}^{(q)} \bar{E}_{m n}^{(r)}-\bar{E}_{k l}^{(q)} \bar{E}_{m n}^{(r)}\right)_{(19)}
\end{aligned}
$$

By substituting the first relation of Eq. (6) and Eq. (19) into Eq. (17), we obtain [11]:

$$
\begin{aligned}
\delta \tilde{V}= & \sum_{n} \bar{T}_{i j}^{(n)} \delta \bar{U}_{i, j}^{(n)}+n \bar{T}_{i j}^{(n-1)} \delta \bar{U}_{i, j}^{(n)} \\
& +\sum_{m, n}\left[\left(\bar{T}_{k j}^{(m+n)} \bar{U}_{i, k}^{(m)}+m \bar{T}_{2 j}^{(m+n-1)} \bar{U}_{i}^{(m)}\right) \delta \bar{U}_{i, j}^{(n)}\right. \\
& +\left(n \bar{T}_{2 j}^{(m+n-1)} \bar{U}_{i, j}^{(m)}\right. \\
& \left.\left.+m n \bar{T}_{22}^{(m+n-2)} \bar{U}_{i}^{(m)}\right) \delta \bar{U}_{i}^{(n)}\right] .
\end{aligned}
$$

By substituting Eqs. (4), (5), (8), and (20) into Eq. (15), using the chain rule of differentiation and the two-dimensional divergence theorem and noting the arbitrariness of the variation in the variational principle, we obtain the $n$ th-order equations of motion:

$$
\begin{aligned}
{\left[\bar{T}_{i j}^{(n)}\right.} & +\sum_{m}\left[\bar{T}_{k j}^{(m+n)} \bar{U}_{i, k}^{(m)}+m \bar{T}_{2 j}^{(m+n-1)} \bar{U}_{i}^{(m)}\right]_{, j} \\
& +n\left[\bar{T}_{2 i}^{(n-1)}+\sum_{m}\left(\bar{T}_{2 j}^{(m+n-1)} \bar{U}_{i, j}^{(m)}\right.\right. \\
& \left.\left.+m \bar{T}_{22}^{(m+n-1)} \bar{U}_{i}^{(m)}\right)\right]+\bar{Q}_{i}^{(n)} \\
& \left.+\rho \sum_{m} a_{(m n)} \bar{B}_{i}^{(m)}=\rho \sum_{m} a_{(m n)} \ddot{u}_{i}^{(m)}\right], \quad \text { in } A
\end{aligned}
$$

with the traction boundary condition of:

$$
\begin{aligned}
n_{j}\left[\bar{T}_{i j}^{(n)}+\right. & \left.\sum_{m}\left(\bar{T}_{k j}^{(m+n)} \bar{U}_{i, k}^{(m)}+m \bar{T}_{2 j}^{(m+n-1)} \bar{U}_{i}^{(m)}\right)\right] \\
& =\bar{P}_{i}^{(n)}, \quad \text { on } C
\end{aligned}
$$

where $n$ th-order components of face traction and edge traction respectively can be defined by $[11,19]$ :

$$
\begin{aligned}
\left(\bar{Q}_{i}^{(n)}, Q_{i}^{(n)}, q_{i}^{(n)}\right)= & {\left[\left[x_{2}^{(n)}\left(\bar{Q}_{i}, Q_{i}, q_{i}\right)\right]_{x_{2}=b}\right.} \\
& \left.+\left[x_{2}^{(n)}\left(\bar{Q}_{i}, Q_{i}, q_{i}\right)\right]_{x_{2}=-b}\right], \\
\left(\bar{P}_{i}^{(n)}, P_{i}^{(n)}, p_{i}^{(n)}\right)= & \int_{-b}^{b} x_{2}^{n}\left(\bar{Q}_{i}, Q_{i}, q_{i}\right) d x_{2} .
\end{aligned}
$$

In Eq. (21), $\ddot{\bar{U}}_{i}^{(n)}$ was replaced with $\ddot{u}_{i}^{(n)}$. The $n$ thorder equation of motion and the traction boundary condition for the final state are the same as the equations obtained in $[11,23]$.

By replacing the barred quantities with unbarred ones in Eqs. (21) and (22), the equation of motion for the initial state is obtained as:

$$
\begin{aligned}
& {\left[T_{i j}^{(n)}+\sum_{m}\left(T_{k j}^{(m+n)} U_{i, k}^{(m)}+m T_{2 j}^{(m+n-1)} U_{i}^{(m)}\right)\right]_{, j}} \\
& \quad-n\left[T_{2 i}^{(n-1)}+\sum_{m}\left(T_{2 j}^{(m+n-1)} U_{i, j}^{(m)}\right.\right. \\
& \left.\left.\quad+m T_{22}^{(m+n-2)} U_{i}^{(m)}\right)\right]+Q_{i}^{(n)} \\
& \quad+\rho \sum_{m} a_{(m n)} B_{i}^{(m)}=0 \quad \text { in } A
\end{aligned}
$$

and the boundary condition is obtained by:

$$
\begin{aligned}
n_{j}\left[T_{i j}^{(n)}+\right. & \left.\sum_{m}\left(T_{k j}^{(m+n)} U_{i, k}^{(m)}+m T_{2 j}^{(m+n-1)} \bar{U}_{i}^{(m)}\right)\right] \\
& =P_{i}^{(n)}, \quad \text { on } C .
\end{aligned}
$$


Also, according to Eq. (19), for the stress in the initial state, we obtain:

$$
\begin{aligned}
T_{i j}^{(p)}= & \sum_{p, q} a_{p q} C_{i j k l}^{\theta}\left(E_{k l}^{(q)}-{E^{\prime}}_{k l}^{(q)}\right) \\
& +\frac{1}{2} \sum_{p, q, r} q_{p q r} C_{i j k l m n}^{\theta}\left(E_{k l}^{(q)} E_{m n}^{(r)}\right. \\
& \left.-{E^{\prime}(q)}_{k l}^{(q)}{E^{\prime}(r)}_{m n}^{(r)}\right) .
\end{aligned}
$$

By subtracting Eqs. (24), (25), and (26) from Eqs. (21), (22), and (19), respectively, and neglecting very small temperature changes from the initial to the final state and products of incremental quantities $[12,15]$, the incremental equation of motion and traction boundary condition are obtained:

$$
\begin{aligned}
& {\left[t_{i j}^{(n)}+\sum_{m}\left(T_{k j}^{(m+n)} u_{i, k}^{(m)}+m T_{2 j}^{(m+n-1)} u_{i}^{(m)}\right.\right.} \\
& \left.\left.\quad+t_{k j}^{(m+n)} U_{i, k}^{(m)}+m t_{2 j}^{(m+n-1)} U_{i}^{(m)}\right)\right]_{, j} \\
& \quad-n\left[t_{2 i}^{(n-1)}+\sum_{m}\left(T_{2 k}^{(m+n-1)} u_{i, k}^{(m)}\right.\right. \\
& \quad+m T_{22}^{(m+n-2)} u_{i}^{(m)}+t_{2 k}^{(m+n-1)} U_{i, k}^{(m)} \\
& \left.\left.\quad+m t_{22}^{(m+n-2)} U_{i}^{(m)}\right)\right]+q_{i}^{(n)}+\rho \sum_{m} a_{(m n)} b_{i}^{(m)} \\
& \quad=\rho \sum_{m} a_{(m n)} \ddot{u}_{i}^{(n)}, \quad \text { in } A, \\
& \left.\left.\quad+t_{j k}^{(m+n)} U_{i, k}^{(m)}+m t_{2 j}^{(m+n-1)} U_{i}^{(m)}\right)\right]=p_{i}^{(n)} \\
& n_{j}\left[t_{i j}^{(n)}+\sum_{m}\left(T_{j k}^{(m+n)} u_{i, k}^{(m)}+m T_{2 j}^{(m+n-1)} u_{i}^{(m)}\right.\right.
\end{aligned}
$$

on $C$,

where:

$$
\begin{aligned}
t_{i j}^{(p)}= & \sum_{q} a_{(p q)} C_{i j k l}^{\theta} \eta_{k l}^{(q)} \\
& +\sum_{q, r} a_{(p q r)} C_{i j k l m n}^{\theta} E_{k l}^{(q)} \eta_{m n}^{(r)} .
\end{aligned}
$$

The equations listed here can be applied to analyze the vibrations of crystal plates. Here, the thickness-shear modes of the crystal plate are of interest; therefore, the first-order equations are derived for the crystal plates in the next section.

\section{First-order incremental equations and frequency changes}

In this article, the effects of the mass and deformation of the electrodes on the resonance frequencies are not considered. Indeed, the electrodes have an impact on the resonance frequencies, but do not affect the frequency changes [19]. To investigate the resonance frequency of thickness-shear vibrations, as mentioned above, it is sufficient to retain terms of order zero and one only for the two-dimensional equations obtained in the previous section $[22,23]$. This means that $U_{i}^{(n)}$, $u_{i}^{(n)}=0$ for $n>1$ and $T_{i j}^{(n)}, t_{i j}^{(n)}, E_{i j}^{(n)}$ and $\eta_{i j}^{(n)}$ are disregarded for $n>1$ [11,19,23,24]. The resulting equations accommodate the coupling of the six lowest modes of vibration, and the strain-displacement relation (i.e., Eq. (6)) reduces to:

$$
\begin{aligned}
E_{i j}^{(0)}= & \frac{1}{2}\left(U_{i, j}^{(0)}+U_{j, i}^{(0)}+\delta_{2 i} U_{j}^{(1)}+\delta_{2 j} U_{i}^{(1)}\right. \\
& +U_{k, i}^{(0)} U_{k, j}^{(0)}+\delta_{2 i} U_{k}^{(1)} U_{k, j}^{(0)}+\delta_{2 j} U_{k}^{(1)} U_{k, i}^{(0)} \\
& \left.+\delta_{2 i} \delta_{2 j} U_{k}^{(1)} U_{k}^{(1)}\right) \\
E_{i j}^{(1)}= & \frac{1}{2}\left(U_{i, j}^{(1)}+U_{j, i}^{(1)}+U_{k, i}^{(0)} U_{k, j}^{(1)}+U_{k, i}^{(1)} U_{k, j}^{(0)}\right. \\
& \left.+\delta_{2 i} U_{k}^{(1)} U_{k, j}^{(1)}+\delta_{2 j} U_{k}^{(1)} U_{k, i}^{(1)}\right) \\
& \left.\left.+u_{k}^{(1)} U_{k, j}^{(1)}\right)+\delta_{2 j}\left(U_{k}^{(1)} u_{k, i}^{(1)}+u_{k}^{(1)} U_{k, i}^{(1)}\right)\right] .(29) \\
\eta_{i j}^{(0)}= & \frac{1}{2}\left[u_{i, j}^{(0)}+u_{j, i}^{(0)}+\delta_{2 i} u_{j}^{(1)}+\delta_{2 j} u_{i}^{(1)}+U_{k, i}^{(0)} u_{k, j}^{(0)}\right. \\
& +U_{k, j}^{(0)} u_{k, i}^{(0)}+\delta_{2 i}\left(U_{k}^{(1)} u_{k, j}^{(0)}+u_{k}^{(1)} U_{k, j}^{(0)}\right) \\
& \left.+\delta_{2 j}\left(U_{k}^{(1)} u_{k, i}^{(0)}+u_{k}^{(1)} U_{k, i}^{(0)}\right)+2 \delta_{2 i} \delta_{2 j} U_{k}^{(1)} u_{k}^{(1)}\right], \\
\eta_{i j}^{(1)}= & \frac{1}{2}\left[u_{i, j}^{(1)}+u_{j, i}^{(1)}+U_{k, i}^{(0)} u_{k, j}^{(1)}+U_{k, j}^{(0)} u_{k, i}^{(1)}+U_{k, j}^{(1)} u_{k, i}^{(0)}+\delta_{2 i}\left(U_{k}^{(1)} u_{k, j}^{(1)}\right.\right. \\
& \\
& \\
& \\
& \\
&
\end{aligned}
$$

By applying Eq. (26), zero and first order initial stresses can be obtained easily by setting $E_{i j}^{(n)},{E^{\prime}}_{k l}^{(n)}=$ 0 for $n>1$ :

$$
T_{i j}^{(0)}=2 b C_{i j k l}^{\theta}\left(E_{k l}^{(0)}-{E^{\prime}}_{k l}^{(0)}\right)
$$




$$
\begin{aligned}
& +b C_{i j k l m n}^{\theta}\left(E_{k l}^{(0)} E_{m n}^{(0)}-{E^{\prime}}_{k l}^{(0)}{E^{\prime}}_{m n}^{(0)}\right) \\
& +\frac{b^{3}}{3} C_{i j k l m n}^{\theta}\left(E_{k l}^{(1)} E_{m n}^{(1)}-{E^{\prime}}_{k l}^{(1)}{E^{\prime}}_{m n}^{(1)}\right), \\
T_{i j}^{(1)}= & \frac{2}{3} b^{3}\left[C_{i j k l}^{\theta}\left(E_{k l}^{(1)}-{E^{\prime}}_{k l}^{(1)}\right)\right. \\
& \left.+C_{i j k l m n}^{\theta}\left(E_{m n}^{(0)} E_{k l}^{(1)}-{E^{\prime}}_{m n}^{(0)}{E^{\prime}}_{k l}^{(1)}\right)\right]
\end{aligned}
$$

Following a method similar to that proposed in $[11,19]$ and by involving some correction factors in the thickness-shear and thickness stretch strains in Eq. (28) and compensating for the approximations in displacement variations of the simple thickness modes $[19,24]$, we obtain:

$$
\begin{aligned}
t_{i j}^{(0)}= & 2 b\left(C_{i j k l}^{\theta}+C_{i j k l m n}^{\theta} E_{m n}^{(0)}\right) k_{(i j)} k_{(k l)} \eta_{k l}^{(0)} \\
& +\frac{2}{3} b^{3} C_{i j k l m n}^{\theta} k_{(i j)} \eta_{k l}^{(1)} E_{m n}^{(1)} \\
t_{i j}^{(1)}= & \frac{2}{3} b^{3} C_{i j k l m n}^{\theta} k_{(i j)} \eta_{k l}^{(0)} E_{m n}^{(1)} \\
& +\frac{2}{3} b^{3}\left(C_{i j k l}^{\theta}+C_{i j k l m n}^{\theta} E_{m n}^{(0)}\right) \eta_{k l}^{(1)}
\end{aligned}
$$

where $k_{(i j)}$ is the correction factor, and we have $k_{(2 i)}=$ $k_{(i 2)}$, and $k_{(i j)}=1$, except $k_{(21)}^{2}=k_{(22)}^{2}=k_{(23)}^{2}=\frac{\pi^{2}}{12}$.

From the first relation of Eq. (27), the equations of motion are, in $A$ :

$$
\begin{aligned}
\left(t_{i j}^{(0)}\right. & +T_{k j}^{(0)} u_{i, k}^{(0)}+T_{k j}^{(1)} u_{i, k}^{(1)}+t_{k j}^{(0)} U_{i, k}^{(0)}+t_{k j}^{(1)} U_{i, k}^{(1)} \\
& \left.+T_{2 j}^{(0)} u_{i}^{(1)}+t_{2 j}^{(0)} U_{i}^{(1)}\right)_{, j}+q_{i}^{(0)}+2 b \rho b_{i}^{(0)} \\
& =2 b \rho \ddot{u}_{i}^{(0)} \\
\left(t_{i j}^{(1)}\right. & \left.+T_{k j}^{(1)} u_{i, k}^{(0)}+t_{k j}^{(1)} U_{i, k}^{(0)}+T_{2 j}^{(1)} u_{i}^{(1)}+t_{2 j}^{(1)} U_{i}^{(1)}\right)_{, j} \\
& -\left(t_{2 j}^{(0)}+T_{2 k}^{(0)} u_{i, k}^{(0)}+T_{2 k}^{(1)} u_{i, k}^{(1)}+t_{2 k}^{(0)} U_{i, k}^{(0)}\right. \\
& \left.+t_{2 k}^{(1)} U_{i, k}^{(1)}+T_{22}^{(0)} u_{i}^{(1)}+t_{22}^{(0)} U_{i}^{(1)}\right)+q_{i}^{(1)} \\
& +\frac{2}{3} b^{3} \rho b_{i}^{(1)}=\frac{2}{3} b^{3} \rho \ddot{u}_{i}^{(1)} .
\end{aligned}
$$

Also, from the second relation of Eq. (27), the traction boundary conditions on $C$ are:

$$
\begin{gathered}
n_{j}\left(t_{i j}^{(0)}+T_{j k}^{(0)} u_{i, k}^{(0)}+T_{j k}^{(1)} u_{i, k}^{(1)}+t_{j k}^{(0)} U_{i, k}^{(0)}+t_{j k}^{(1)} U_{i, k}^{(1)}\right. \\
\left.+T_{2 j}^{(0)} u_{i}^{(1)}+t_{2 j}^{(0)} U_{i}^{(1)}\right)=p_{i}^{(0)}, \\
n_{j}\left(t_{i j}^{(1)}+T_{j k}^{(1)} u_{i, k}^{(0)}+t_{j k}^{(1)} U_{i, k}^{(0)}+T_{2 j}^{(1)} u_{i}^{(1)}+t_{2 j}^{(1)} U_{i}^{(1)}\right) \\
=p_{i}^{(1) .}
\end{gathered}
$$

For convenience, the abbreviated (Voigt) notation is employed. On this basis, a pair of indices like $p q$ is replaced with a single index according to $11 \rightarrow 1,22 \rightarrow$ $2,33 \rightarrow 3,23 \rightarrow 4,13 \rightarrow 5,21 \rightarrow 6$. The equation of motion, Eq. (32), may be written in six expanded forms, two of which are important to us:

$$
\begin{aligned}
& \frac{\partial}{\partial x_{1}}\left[U_{2,1}^{(0)} t_{1}^{(0)}+U_{2,3}^{(0)} t_{5}^{(0)}+\left(1+U_{2}^{(1)}\right) t_{6}^{(0)}+U_{2,1}^{(1)} t_{1}^{(1)}\right. \\
& +U_{2,3}^{(1)} t_{5}^{(1)}+T_{1}^{(0)} u_{2,1}^{(0)}+T_{5}^{(0)} u_{2,3}^{(0)} \\
& \left.+T_{1}^{(1)} u_{2,1}^{(1)}+T_{5}^{(1)} u_{2,3}^{(1)}+T_{6}^{(0)} u_{2}^{(1)}\right] \\
& +\frac{\partial}{\partial x_{3}}\left[U_{2,1}^{(0)} t_{5}^{(0)}+U_{2,3}^{(0)} t_{3}^{(0)}\right. \\
& +\left(1+U_{2}^{(1)}\right) t_{4}^{(0)}+U_{2,1}^{(1)} t_{5}^{(1)}+U_{2,3}^{(1)} t_{3}^{(1)} \\
& +T_{5}^{(0)} u_{2,1}^{(0)}+T_{3}^{(0)} u_{2,3}^{(0)}+T_{5}^{(1)} u_{2,1}^{(1)} \\
& \left.+T_{3}^{(1)} u_{2,3}^{(1)}+T_{4}^{(0)} u_{2}^{(1)}\right]+q_{2}^{(0)}+2 b \rho b_{2}^{(0)} \\
& =2 b \rho \ddot{u}_{2}^{(0)}, \\
& \frac{\partial}{\partial x_{1}}\left[\left(1+U_{1,1}^{(0)}\right) t_{1}^{(1)}+U_{1,3}^{(0)} t_{5}^{(1)}+U_{1}^{(1)} t_{6}^{(1)}+T_{1}^{(1)} u_{1,1}^{(0)}\right. \\
& \left.+T_{5}^{(1)} u_{1,3}^{(0)}+T_{6}^{(1)} u_{1}^{(1)}\right]+\frac{\partial}{\partial x_{3}}\left[\left(1+U_{1,1}^{(0)}\right) t_{5}^{(1)}\right. \\
& +U_{1,3}^{(0)} t_{3}^{(1)}+U_{1}^{(1)} t_{4}^{(1)}+T_{5}^{(1)} u_{1,1}^{(0)}+T_{3}^{(1)} u_{1,3}^{(0)} \\
& \left.+T_{4}^{(1)} u_{1}^{(1)}\right]-\left[\left(1+U_{1,1}^{(0)}\right) t_{6}^{(0)}+U_{1,3}^{(0)} t_{4}^{(0)}\right. \\
& +U_{1}^{(1)} t_{2}^{(0)}+U_{1,1}^{(1)} t_{6}^{(1)}+U_{1,3}^{(1)} t_{4}^{(1)}+T_{6}^{(0)} u_{1,1}^{(0)} \\
& \left.+T_{4}^{(0)} u_{1,3}^{(0)}+T_{6}^{(1)} u_{1,1}^{(1)}+T_{4}^{(1)} u_{1,3}^{(1)}+T_{2}^{(0)} u_{1}^{(1)}\right] \\
& +q_{1}^{(1)}+\frac{2}{3} b^{3} \rho b_{1}^{(1)}=\frac{2}{3} b^{3} \rho \ddot{u}_{1}^{(1)} .
\end{aligned}
$$


The equations of motion have a similar form to those obtained by Lee et al. [11], and the homogenous steady thermal strains do not change the form of the equation of motion, though these strains change the initial strains, displacement gradients, and the incremental stresses.

\section{Resonance frequency shift of rotated $Y$-cut quartz due to initial thermal and mechanical strains}

In this section, the change in the resonance frequency of a rotated Y-cut quartz crystal, vibrating predominately in the thickness-shear mode, is evaluated by using the equations obtained in the last section.

In Y-cut crystals, $x_{1}$ is the diagonal axis in $x_{1}$ and $x_{3}$ planes of the plate, and some of the secondand third-order elastic coefficients are zero. For rotated Y-cut, we have:

$$
\begin{aligned}
& C_{p q}=0, \quad p=1,2,3,4 \text { and } q=5,6, \\
& C_{p q r}=0, \quad p, q=1,2,3,4 \text { and } r=5,6, \\
& \text { or } \quad p, q, r=5,6 .
\end{aligned}
$$

In this article, the measured values of the secondorder elastic stiffness coefficient by Bechmann [25], the temperature derivatives of the second-order elastic stiffness by Lee and Yong [26], and the third-order elastic stiffness coefficients by Thurston et al. [27] are used.

We consider a circular disc of rotated Y-cut that is subjected to a uniform temperature change. Then, two opposed diametric forces are applied to the disc. This condition induces in-plane stress components $T_{1}^{(0)}$, $T_{3}^{(0)}$, and $T_{5}^{(0)}$ that only depend on the diametric forces; all other components of stress can be disregarded [11]. The values of the initial stresses can be computed by applying Eq. (30).

In calculating the values of the initial stresses, Lee [15] linearized the stress equation without a significant decrease in accuracy. In our case, due to the insertion of thermal strains into Eq. (30), the effect of linearization on the accuracy should be verified. Assuming that the change in temperature of the initial state is less than $100^{\circ} \mathrm{C}$ in the $\mathrm{Y}$-cut quartz, the thermal strains can be calculated from Eq. (36) [14]:

$$
E^{\prime(0)}=\alpha_{i j}^{\theta} \Delta T
$$

where $\alpha_{i j}^{\theta}$ is the thermal expansion coefficients, measured by Bechmann [25]. For Y-cut quartz crystals, only ${E^{\prime}}_{1}^{(0)},{E^{\prime}}_{2}^{(0)}$, and ${E^{\prime}}_{3}^{(0)}$ are non-zero. These strain components are calculated, and the effect of nonlinear terms on Eq. (30) is found to be less than 2 percent of the linear terms at $100^{\circ} \mathrm{C}$. The error decreases rapidly with lowering of the temperature. Thus, with a high degree of accuracy, the initial stresses can be calculated using this equation:

$$
T_{i j}^{(0)}=2 b C_{i j k l}\left(E_{k l}^{(0)}-{E^{\prime}}_{k l}^{(0)}\right)
$$

where $E_{k l}^{(0)}$ is the sum of the thermal and mechanical strains [12]. The thermal strain, $E_{k l}^{\prime(0)}$, compensates for the effect of the thermal strain involved in $E_{k l}^{(0)}$. Thus, with a high degree of accuracy, the initial stress is only induced by mechanical strains.

Setting $U_{i}^{(n)}, u_{i}^{(n)}=0$ for $n>1$ in the first relation of $\mathrm{Eq}$. (6) and disregarding the products of displacement gradient, the initial strain components can be obtained. If the initial displacement gradients are only due to application of diametric forces, then the components of $U_{2,1}^{(0)}$ and $U_{2,3}^{(0)}$ can be set to zero [11]. Nevertheless, in our problem with an initial thermal loading, these components cannot be disregarded. These displacement gradient components affect $E_{4}^{(0)}$ and $E_{6}^{(0)}$; thus, we have:

$$
\begin{array}{ll}
E_{1}^{(0)}=U_{1,1}^{(0)}, & E_{2}^{(0)}=U_{2,2}^{(0)} \\
E_{3}^{(0)}=U_{3,3}^{(0)}, & E_{4}^{(0)}=U_{2,3}^{(0)}+U_{3}^{(1)} \\
E_{5}^{(0)}=U_{3,1}^{(0)}+U_{1,3}^{(0)}, & E_{6}^{(0)}=U_{2,1}^{(0)}+U_{1}^{(1)} .
\end{array}
$$

By assuming flexural and thickness-shear vibrations, which are predominant in the $x_{1}$ direction and are independent of $x_{3}$, the components of $u_{1}^{(1)}$ and $u_{2}^{(0)}$ are functions of $t$ and $x_{1}$. All other components of the incremental displacements are set to zero. The nonzero incremental strain components are:

$$
\begin{aligned}
& \eta_{2}^{(0)}=U_{1}^{(1)} u_{1}^{(1)} \\
& \eta_{4}^{(0)}=U_{1,3}^{(0)} u_{1}^{(1)} \\
& \eta_{6}^{(0)}=\left(1+U_{2}^{(1)}\right) u_{2,1}^{(0)}+\left(1+U_{1,1}^{(0)}\right) u_{1}^{(1)}, \\
& \eta_{1}^{(1)}=\left(1+U_{1,1}^{(0)}\right) u_{1,1}^{(1)}, \\
& \eta_{5}^{(1)}=U_{1,3}^{(0)} u_{1,1}^{(1)}+U_{2,3}^{(0)} u_{2,1}^{(1)}, \\
& \eta_{6}^{(1)}=U_{1}^{(1)} u_{1,1}^{(1)},
\end{aligned}
$$

which differ in $\eta_{6}^{(0)}$ and $\eta_{5}^{(1)}$ from the similar strain components which were derived for pure mechanical problem in [11]. Consequently, by substituting the 
initial and incremental strains into Eq. (34), the displacement equations of motion can be obtained without face tractions and body forces:

$$
\begin{gathered}
\frac{\partial}{\partial x_{1}}\left[U_{2,3}^{(0)} t_{5}^{(0)}+\left(1+U_{2}^{(1)}\right) t_{6}^{(0)}+T_{1}^{(0)} u_{2,1}^{(0)}\right]=2 b \rho \ddot{u}_{2}^{(0)}, \\
\frac{\partial}{\partial x_{1}}\left[\left(1+U_{1,1}^{(0)}\right) t_{1}^{(1)}+U_{1,3}^{(0)} t_{5}^{(1)}+U_{1}^{(1)} t_{6}^{(1)}\right] \\
-\left(1+U_{1,1}^{(0)}\right) t_{6}^{(0)}-U_{1,3}^{(0)} t_{4}^{(0)}-U_{1}^{(1)} t_{2}^{(0)} \\
=\frac{2}{3} b^{3} \rho \ddot{u}_{1}^{(1)},
\end{gathered}
$$

where the first equation is related to the flexural vibrations, and the second equation is related to the thickness-shear vibrations. This result shows that the flexural motion equation differs from the equation proposed by Lee for pure mechanical problem, but the thickness-shear equation is similar. From Eq. (31), the zeros and first-order incremental stresses are [11,19,23]:

$$
\begin{aligned}
& t_{p}^{(0)}=2 b k^{\mu+v}\left(C_{p q}^{\theta}+C_{p q r}^{\theta} E_{r}^{(0)}\right) \eta_{q}^{(0)}, \quad q=2,4,6 \\
& t_{p}^{(1)}=\frac{2 b^{3}}{3}\left(C_{p q}^{\theta}+C_{p q r}^{\theta} E_{r}^{(0)}\right) \eta_{q}^{(1)}, \quad q=1,5,6,
\end{aligned}
$$

where $k=k_{(21)}=k_{(22)}=k_{(23)}=\pi / \sqrt{12}$, and we have [23]:

$$
\mu=\cos ^{2}\left(\frac{p \pi}{2}\right), \quad v=\cos ^{2}\left(\frac{q \pi}{2}\right) .
$$

By substituting Eqs. (38) and (39) into Eqs. (41), and then into Eq. (40), the flexural and thickness-shear time-displacement equations can be obtained:

$$
\begin{gathered}
\left(k^{2}\left[C_{66}^{\theta}\left(1+2 U_{2}^{(1)}\right)+C_{66 r}^{\theta} E_{r}^{(0)}\right]+\frac{1}{2 b} T_{1}^{(0)}\right. \\
\left.+U_{2,3}^{(0)} C_{56}^{\theta}\right) u_{2,11}^{(0)}+k^{2}\left(C _ { 6 6 } ^ { \theta } \left[1+U_{1,1}^{(0)}\right.\right. \\
\left.\left.+U_{2}^{(1)}+C_{66 r}^{\theta} E_{r}^{(0)}\right]+U_{2,3}^{(0)} C_{56}^{\theta}\right) u_{1,1}^{(1)} \\
=\rho \ddot{u}_{2}, \\
{\left[C_{11}^{\theta}\left(1+U_{1,1}^{(0)}\right)+C_{11 r}^{\theta} E_{r}^{(0)}\right] u_{1,11}^{(1)}-\frac{3}{b^{2}} k^{2}\left(\left[C_{66}^{\theta}(1\right.\right.} \\
\left.\left.+U_{1,1}^{(0)}+U_{2}^{(1)}\right)+C_{66 r}^{\theta} E_{r}^{(0)}\right] u_{2,1}^{(0)} \\
\left.+\left[C_{66}^{\theta}\left(1+2 U_{1,1}^{(0)}\right)+C_{66 r}^{\theta} E_{r}^{(0)}\right] u_{1}^{(1)}\right)=\rho \ddot{u}_{1}^{(1)} \\
(43)
\end{gathered}
$$

Assume the straight-crested wave propagated in the $x_{1}$ direction to be $[11,19]$ :

$$
\begin{aligned}
& u_{1}^{(1)}=A_{1}^{(1)} e^{i\left(\xi x_{1}-\omega t\right)}, \\
& u_{2}^{(0)}=-i A_{2}^{(0)} b e^{i\left(\xi x_{1}-\omega t\right)},
\end{aligned}
$$

where $A_{1}^{(1)}$ and $A_{2}^{(0)}$ are the wave amplitudes, and $\zeta$ is the wave number.

By substituting Eq. (44) into Eqs. (43), the frequency equation can be obtained:

$$
\begin{aligned}
\Omega^{2}= & \frac{1}{2}\left(M_{2}+\left(M_{1}+M_{4} / 3\right) z^{2} \pm\left(\left[M_{2}+\left(M_{1}\right.\right.\right.\right. \\
& \left.\left.\left.\left.-\frac{M_{4}}{3}\right) z^{2}\right]^{2}+\frac{4 M_{3}^{2} z^{2}}{3}\right)^{\frac{1}{2}}\right),
\end{aligned}
$$

where:

$$
\begin{aligned}
& M_{1}=\left[\left(1+2 U_{1,1}^{(0)}\right) C_{11}^{\theta} / C_{66}^{\theta}+C_{11 r}^{\theta} E_{r}^{(0)} / C_{66}^{\theta}\right] / 3 k^{2}, \\
& M_{2}=1+2 U_{1,1}^{(0)}+C_{66 r}^{\theta} E_{r}^{(0)} / c_{66}^{\theta}, \\
& M_{3}=1+U_{1,1}^{(0)}+U_{2}^{(1)}+\frac{C_{66 r}^{\theta} E_{r}^{(0)}}{C_{66}^{\theta}}+\frac{C_{56}^{\theta} U_{1,1}^{(1)}}{k^{2} C_{66}^{\theta}}, \\
& M_{4}=1+2 U_{2}^{(1)}+\frac{C_{66 r}^{\theta} E_{r}^{(0)}}{C_{66}^{\theta}}+\frac{T_{1}^{(0)}}{2 b k^{2} C_{66}^{\theta}}+\frac{C_{56} U_{2,3}^{(0)}}{k^{2} C_{66}^{\theta}}, \\
& \Omega=\omega / \omega_{1}, \quad z=\xi b, \\
& \omega_{1}=\left[\frac{3 k^{2} C_{66}^{\theta}}{\rho b^{2}}\right]^{1 / 2},
\end{aligned}
$$

where $\omega_{1}$ is the lowest thickness-shear cut-off frequency of the quartz without initial stress $[11,19]$. For $|z|<<1$ and $|z|<<\Omega$, the terms associated with $z^{4}$ can be disregarded in Eq. (45), and by using the Taylor expansion and keeping the first two terms only, the frequency equation can be obtained:

$$
\Omega^{2}=M_{2}+\left(M_{1}+M_{3}^{2} / 3 M_{2}\right) z^{2},
$$

which leads to the cut-off frequency of thickness-shear vibration by setting $z=0$ :

$$
\Omega^{2}=1+2 U_{1,1}^{(0)}+\frac{C_{66 r}^{\theta} E_{r}^{(0)}}{C_{66}^{\theta}} .
$$

Frequency Eq. (48) gives the resonance frequency of thickness-shear mode of vibration in the rotated $\mathrm{Y}$ cut quartz, which is under homogenous initial thermal and mechanical strains. This equation is identical to Lee's, which is obtained for initial mechanical stresses 
only. The similarity shows that the initial steady homogenous thermal strains do not change the form of the frequency equation in thickness-shear mode. Eq. (48) for an AT-cut quartz disc can be rewritten as:

$$
\begin{aligned}
\frac{\Delta f}{f_{0}}= & \Omega-1=U_{1,1}^{(0)}+\frac{1}{2 C_{66}^{\theta}}\left[C_{661}^{\theta} E_{1}^{(0)}+C_{662}^{\theta} E_{2}^{(0)}\right. \\
& \left.+C_{663}^{\theta} E_{3}^{(0)}+C_{664}^{\theta} E_{4}^{(0)}\right],
\end{aligned}
$$

where strain components include both mechanical and thermal strains. Using Eq. (49), the force-frequency coefficient of rotated Y-cut quartz can be predicted at various temperatures. The force-frequency coefficient at any temperature is related to the frequency shift arising from the application of opposing diametric forces at that temperature. Since all the initial strains and displacement gradients, as mentioned in the previous section, are the sum of mechanical and thermal strains and gradients, Eq. (49) can be rewritten in the form of:

$$
\begin{aligned}
\frac{\Delta f}{f_{0}}= & \Omega-1=\left(U_{1,1}^{(0)}\right)_{m}+\left(U_{1,1}^{(0)}\right)_{T} \\
& +\frac{1}{2 C_{66}^{\theta}}\left[C_{661}^{\theta}\left(\left(E_{1}^{(0)}\right)_{m}+\left(E_{1}^{(0)}\right)_{T}\right)\right. \\
& +C_{662}^{\theta}\left(\left(E_{2}^{(0)}\right)_{m}+\left(E_{2}^{(0)}\right)_{T}\right) \\
& +C_{663}^{\theta}\left(\left(E_{3}^{(0)}\right)_{m}+\left(E_{3}^{(0)}\right)_{T}\right) \\
& \left.+C_{664}^{\theta}\left(\left(E_{4}^{(0)}\right)_{m}+\left(E_{4}^{(0)}\right)_{T}\right)\right] \\
& =\left(\frac{\Delta f}{f_{0}}\right)_{\text {mechanical }}+\left(\frac{\Delta f}{f_{0}}\right)_{\text {thermal }},
\end{aligned}
$$

where $\frac{\Delta f}{f_{0}}$ is the frequency shift due to the application of both initial mechanical and thermal strains; $\left(E_{i}\right)_{m}$ and $\left(U_{1,1}^{(0)}\right)_{m}$ are the mechanical strain and displacement gradient. Also, $\left(E_{i}\right)_{T}$ and $\left(U_{1,1}^{(0)}\right)_{T}$ are the thermal strain and displacement gradient.

The force-frequency effect, at a definite temperature as mentioned above, is related to the mechanical strains only. Due to linear superposition of strains in Eq. (50), both mechanical and thermal strains have distinct effects on frequency. Nevertheless, to obtain the force-frequency coefficient in quartz, only the mechanical strains at the working temperature are involved. By taking into account only the first temperature derivative of TOEs, we have [21]:

$$
C_{66 i}^{\theta}=C_{66 i}^{25^{\circ} \mathrm{C}}+\tilde{C}_{66 i} \times \Delta T,
$$

where $C_{66 i}^{25^{\circ} \mathrm{C}}$ is the value of $C_{66 i}$ at temperature of $25^{\circ} \mathrm{C}$, the coefficient $\tilde{C}_{66 i}$ is the first temperature derivative of the coefficient $C_{66 i}$, and $\Delta T=(\theta-25)^{\circ} \mathrm{C}$, where $\theta$ is the final temperature of the disk. The mechanical version of Eq. (50) is related to the forcefrequency effect. Thus, by substituting Eq. (51) into Eq. (50), for mechanical version of Eq. (50), we obtain:

$$
\begin{aligned}
\left(\frac{\Delta f}{f_{0}}\right)_{m}= & \left(U_{1,1}^{(0)}\right)_{m}+\frac{1}{2 C_{66}^{\theta}}\left[\left(C_{661}^{25^{\circ} \mathrm{C}}+\tilde{C}_{661}\right.\right. \\
& \times \Delta T)\left(E_{1}^{(0)}\right)_{m}+\left(C_{662}^{25^{\circ} \mathrm{C}}+\tilde{C}_{662}\right. \\
& \times \Delta T)\left(E_{2}^{(0)}\right)_{m}+\left(C_{663}^{25^{\circ} \mathrm{C}}+\tilde{C}_{663}\right. \\
& \times \Delta T)\left(E_{3}^{(0)}\right)_{m}+\left(C_{664}^{25^{\circ} \mathrm{C}}+\tilde{C}_{664}\right. \\
& \left.\times \Delta T)\left(E_{4}^{(0)}\right)_{m}\right] .
\end{aligned}
$$

\section{Results and discussion}

As mentioned in the introduction, due to the forcefrequency effect, the resonance frequency of piezoelectric resonators will change in proportion to a pair of external opposite diametrical forces applied at the rim of the resonator [19]. The force-frequency effect can be quantified by force-the frequency coefficient, $K(\psi)$, which is defined by [28]:

$$
\frac{\Delta f}{f_{0}}=K(\psi) \frac{F(\psi) N}{2 b D},
$$

where $F(\psi)$ is the applied force with azimuth angle of $\psi, \Delta f / f_{0}$ is the fractional frequency shift, $N$ is the frequency constant, and $D$ is the plate diameter, and $2 b$ is its thickness.

\subsection{Calculating the force-frequency constants at $25^{\circ} \mathrm{C}$ for circular disks}

The force-frequency coefficient for singly and doubly rotated quartz resonators was measured previously at room temperature [29]. To measure the force-frequency coefficients, a circular plate of quartz is subjected to a pair of diametric forces at a constant uniform temperature. The forces make an angle of $\psi$ (azimuth angle) with $x_{1}$ axis, as shown in Figure 4 . In these circumstances, the initial stress field is not homogenous.

Some researchers $[28,11]$ have used the uniform stress solution to obtain the strain components by using an anisotropic constitutive model. They showed that such an approach closely matches the force-frequency experiments. Based on this approach, the stress distributions for the resonator with a diameter of $D$ 


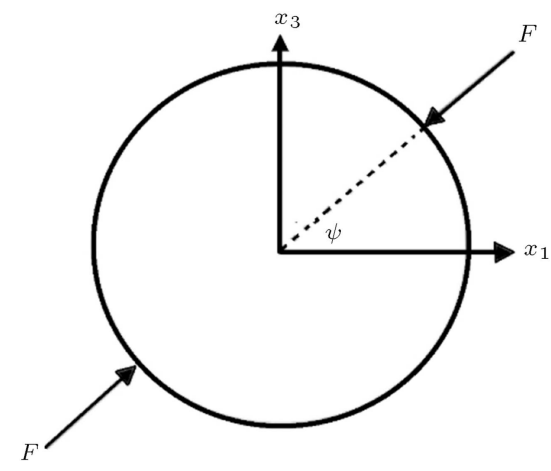

Figure 4. Circular quartz disc subjected to two opposed diametric forces.

and thickness of $2 b$, when is subjected to a pair of diametrical forces, can be obtained as:

$$
\begin{aligned}
& T_{1}=\frac{-2}{\pi} \frac{F}{2 b D}(1+2 \cos 2 \psi), \\
& T_{3}=\frac{-2}{\pi} \frac{F}{2 b D}(1-2 \cos 2 \psi), \quad T_{5}=\frac{-4}{\pi} \frac{F}{2 b D} \sin 2 \psi
\end{aligned}
$$

Then, the strain components for rotated Y-cut of quartz can be obtained as follows:

$$
\begin{aligned}
& E_{1}=S_{11}^{\theta} T_{1}+S_{13}^{\theta} T_{3}+S_{15}^{\theta} T_{5}, \\
& E_{2}=S_{21}^{\theta} T_{1}+S_{23}^{\theta} T_{3}+S_{25}^{\theta} T_{5}, \\
& E_{3}=S_{31}^{\theta} T_{1}+S_{33}^{\theta} T_{3}+S_{35}^{\theta} T_{5}, \\
& E_{4}=S_{41}^{\theta} T_{1}+S_{43}^{\theta} T_{3}+S_{45}^{\theta} T_{5},
\end{aligned}
$$

where $S_{i j}^{\theta}$ is the compliance of AT-Cut quartz at temperature $\theta$. From Eq. (19) and by neglecting higher-order components of stresses, we obtain $[11,28]$ :

$$
T_{i}^{(0)}=2 b T_{i}=2 b C_{i j} E_{j}^{(0)} .
$$

Eq. (55) gives the components of strains due to mechanical load, which are necessary for calculating the frequency shift with Eq. (52). By substituting Eq. (52) into Eq. (53), the force-frequency coefficient at a constant uniform temperature can be obtained:

$$
\begin{aligned}
K^{\theta}(\psi)= & \frac{1}{2 b^{\theta} f_{0}}\left[U_{1}^{(0)}(\psi)+\frac{1}{2 C_{66}^{\theta}}\left(\left(C_{661}^{25^{\circ} \mathrm{C}}+\tilde{C}_{661}\right.\right.\right. \\
& \times \Delta T)\left(E_{1}^{(0)}\right)_{m}+\left(C_{662}^{25^{\circ} \mathrm{C}}+\tilde{C}_{662} \times \Delta T\right) \\
& \left(E_{2}^{(0)}\right)_{m}+\left(C_{663}^{25^{\circ} \mathrm{C}}+\tilde{C}_{663} \times \Delta T\right)\left(E_{3}^{(0)}\right)_{m} \\
& \left.\left.+\left(C_{664}^{25^{\circ} \mathrm{C}}+\tilde{C}_{664} \times \Delta T\right)\left(E_{4}^{(0)}\right)_{m}\right)\right] .
\end{aligned}
$$

Eq. (57) gives the force-frequency coefficient for any

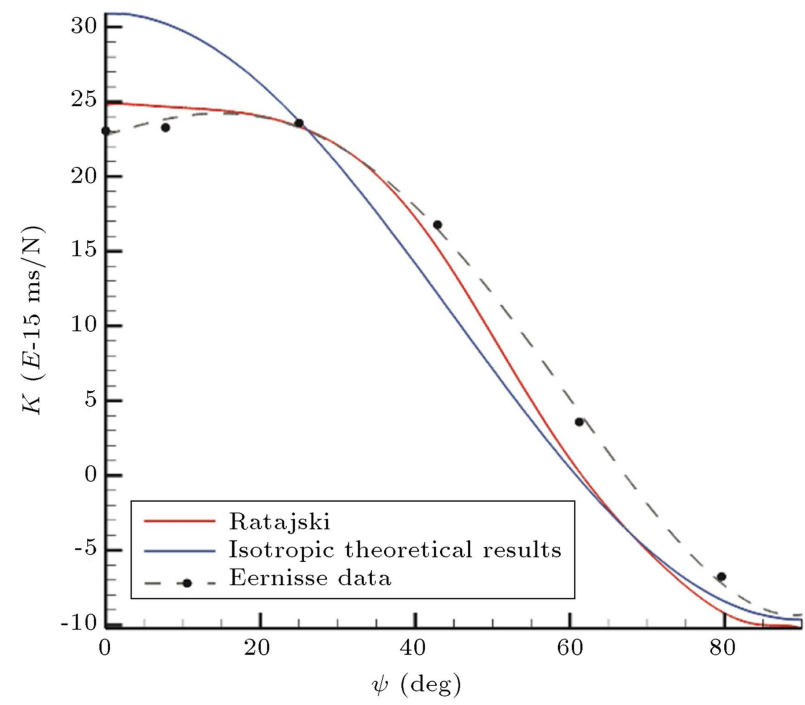

Figure 5. Theoretical and experimental force-frequency coefficients at $25^{\circ} \mathrm{C}$.

azimuth angle at the working temperature. Using this equation and the second- and third-order elastic stiffness coefficients for AT-cut, as published in [30], the force-frequency coefficients at room temperature are calculated as a function of the azimuth angle.

To calculate force-frequency constants at room temperature by Eq. (57), we considered a circular ATcut quartz disc with a diameter of $6 \mathrm{~mm}$, thickness of $0.1660 \mathrm{~mm}$, and fundamental first thickness-shear frequency of $10 \mathrm{MHz}$ subjected to two diametrically opposed forces. Figure 5 shows the calculated forcefrequency constants as a function of azimuth angle in comparison with the experimental results obtained by Ratajskey and EerNisse $[9,30]$. It should be mentioned that our model produces similar results to the Lee's model at room temperature.

To increase the accuracy of the force-frequency curve, the initial stress field is solved by finite element method for quartz disc with anisotropic characteristics and non-uniform stress distribution. Since the quartz crystals have anisotropic characteristics, their governing equations are non-linear, and they cannot be implemented using the default feature of the FEA software. Therefore, the whole governing equations and the boundary conditions are converted to weakform expressions. So, the anisotropic characteristics of quartz can be implemented using the PDE (Partial Differential Equation) interference of the software. Our model includes 2094 Lagrangian quadratic elements. As the thickness-shear mode is important, in the investigation of the force-frequency effect, a swept mesh is utilized to control the mesh number in the thickness direction. The three-dimensional governing equations for quartz, adopted in FEM model, are [19,23]: 


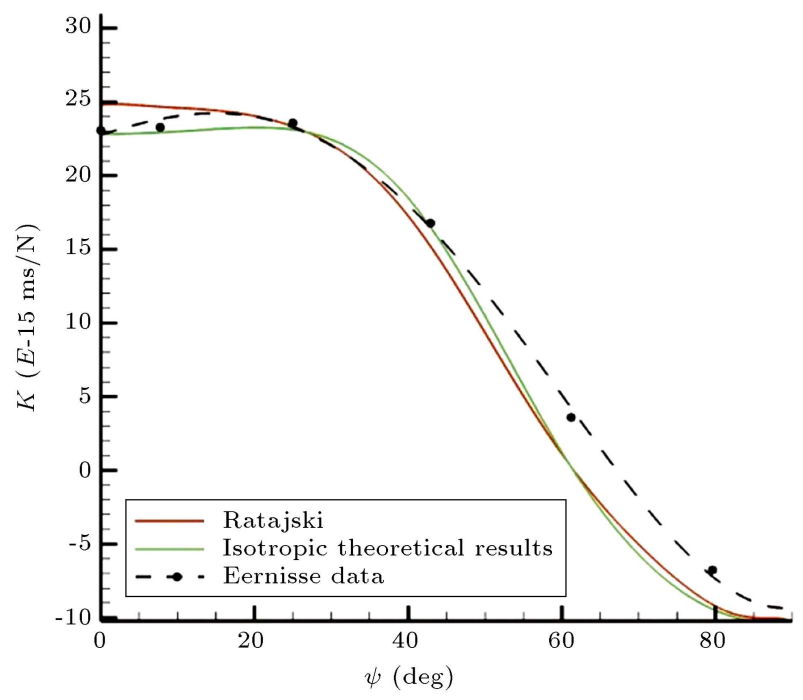

Figure 6. Force-frequency curve based on anisotropic assumptions in calculating the initial stresses.

$$
T_{i j}=C_{i j k l}^{\theta} E_{k l}+\frac{1}{2} C_{i j k l m n}^{\theta} E_{k l} E_{m n}-\lambda_{i j}^{\theta},
$$

and:

$$
\begin{aligned}
& \left(T_{i j}+T_{j k} U_{i, k}\right)_{, j}=0 \text { in } V, \\
& P_{i}=n_{j}\left(T_{i j}+T_{j k} U_{i, k}\right) \text { on } S .
\end{aligned}
$$

The initial strain components are obtained by the second relation of Eq. (1).

The calculated values for non-uniform stresses at the center of the disk are substituted into Eq. (59) to calculate the new force-frequency constants as a function of azimuth angle. Figure 6 depicts the new force-frequency curve in comparison with experimental data.

Consideration of the anisotropy in determining the initial stress bias can enhance the accuracy of the force-frequency model, especially at azimuth angle between $0^{\circ}$ and $25^{\circ}$, as is depicted in Figure 6 [31].

\subsection{Calculating the force-frequency constants at $78^{\circ} \mathrm{C}$}

Analytical calculation of the force-frequency coefficients of quartz at higher temperatures has not been carried out up to now. Experimental values of the force-frequency coefficient of circular AT-cut quartz crystal at temperatures of $78^{\circ} \mathrm{C}$ are available [30] Based on Eq. (57), variations in plate thickness, the second-order elastic stiffness and compliance, and TOEs can lead to the variations in the force-frequency coefficient. Among these parameters, only the temperature derivatives of the TOEs are still unknown. The second-order elastic compliance and stiffness coefficients can be calculated using the temperature derivatives of the elasticity coefficient obtained by Yong and Lee [26]. The thickness of the plate at

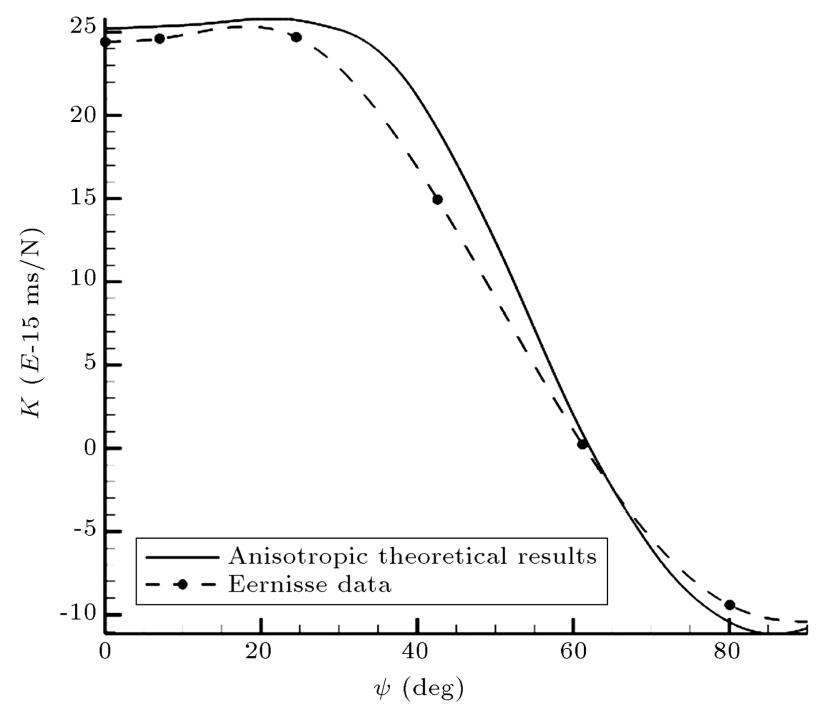

Figure 7. Analytical calculation of force-frequency curve at $78^{\circ} \mathrm{C}$ based on non-uniform stress assumption.

$78^{\circ} \mathrm{C}$ can be determined using the thermal expansion coefficient for AT-cut [25]. By applying the calculated material constants at $78^{\circ} \mathrm{C}$, the initial stress and strain fields are solved both analytically with uniform stress assumption and numerically with non-uniform stress assumption as mentioned in the previous section. Then, the force-frequency constants are calculated using Eq. (57). In this calculation, we used the room temperature values of TOEs measured by Thurston [27] and neglected their temperature derivatives, which are steel unknown to us. Figure 7 shows the calculated and experimental force-frequency curve at $78^{\circ} \mathrm{C}$ for AT-cut quartz crystals.

As seen in Figure 7, the force-frequency curve obtained by analytical model is close to the experimental data at $78^{\circ} \mathrm{C}$.

Performing the force-frequency experiments at high temperatures is a hard task, and there are a few experimental data on the force-frequency effect of the crystal resonators at high temperatures. The developed mathematical model can be utilized in calculating the temperature dependence of the force-frequency effect for rotated Y-Cut quartz resonators. This gives us higher abilities in designing and optimizing the quartz resonators and sensors which may work at various temperatures. For example, as can be seen in Figure 7 , based on the experimental data and analytical model, at azimuth angles near $\psi=60^{\circ}$, the force-frequency coefficient approaches zero. On this basis, for ATCut quartz resonators working at temperatures near $78^{\circ} \mathrm{C}$, it is suggested to connect the resonator holders to the resonator by this azimuth angle. This reduces the acceleration and vibration sensitivity of the resonator and enhances its frequency stability. On the other hand, based on the analytical model and experimental data, the highest force sensitivity of the resonator 
occurs near the azimuth angle $\psi=20^{\circ}$. Thus, this azimuth angle can be chosen for achieving the highest force sensitivity in resonator load cells and some of the resonator pressure sensors which are working at the mentioned temperature.

\section{Conclusion}

In this research, the effect of initial homogenous thermal strains on the force-frequency effect of quartz crystal plates was investigated. We extracted twodimensional equations for high-frequency vibrations of the crystal plates under initial mechanical stresses and homogenous thermal strains by adopting the Mindlin procedure. These equations are general and can be employed for analyzing the vibration of crystal plates with weak piezoelectricity, like quartz, under homogenous thermal and mechanical biasing fields. The derived equations were solved for rotated Y-cut quartz crystal, and an explicit formula was derived for calculating the resonance frequency shift in the thickness-shear mode of the crystal due to mechanical and homogenous thermal biases. By using this formula, the force-frequency curve for a circular AT-Cut disc at $78^{\circ} \mathrm{C}$ is obtained. The calculated force-frequency curve was in good agreement with the experimental data. The developed model can be used for more accurate prediction of quartz crystal vibrations under thermomechanical strains with vast applications in the design and testing of pressure sensors for down-hole usage.

\section{References}

1. Yong, Y.K., Patel, M.S. and Tanaka, M. "Theory and experimental verifications of the resonator $Q$ and equivalent electrical parameters due to viscoelastic and mounting supports losses", IEEE Trans. Ultrason., Ferroelectr., Freq. Control., 57(8), pp. 1831-1839 (2010).

2. Chen, G., Wu, R., Wang, J., Du, J. and Yang, J. "Five-mode frequency spectra of $\mathrm{x}$ 3-dependent modes in AT-cut quartz resonators", IEEE Trans. Ultrason., Ferroelectr., Freq. Control., 59(4), pp. 811-816 (2012).

3. Xie, X., Kong, L., Wang, Y., Zhang, J. and Hu, $Y$. "Coupled vibrations and frequency shift of compound system consisting of quartz crystal resonator in thickness-shear motions and micro-beam array immersed in liquid", Appl. Math. and Mech., 36(2), pp. 225-232 (2015).

4. Zhang, H., Turner, J.A. and Kosinski. J.A. "Experimental measurements of the force-frequency effect of thickness-mode langasite resonators", IEEE Trans. Ultrason., Ferroelectr., Freq. Control., 60(7), pp. 14751478 (2013).

5. Hatipoglu, G. and Tadigadapa, S. "Experimental studies in magnetically induced transverse force-frequency effect in thin quartz microresonators", J. Appl. Phys., 118(3), p. 034508 (2015).

6. Uchiho, T., Uzuka, K., Suenaga, H. and Morita, T. "Quartz force sensor and facing spiral coils for noncontact detection", Jpn. J. Appl. Phys., 53(7S), pp. 07KD09-1-07KD09-4 (2014).

7. Goujon, N., Hori, H., Liang, K.K. and Sinha, B.K. "Applications of piezoelectric materials in oilfield services", IEEE Trans. Ultrason., Ferroelectr., Freq. Control., 59(9), pp. 2042-2050 (2012).

8. CUI, D., Wang, Q., Tao, M. and Zhou., H. "Development of quartz crystal soil pressure sensor with three components", Piezoelectrics \& Acoustooptics, 3 (2013).

9. Ratajski, J.M. "Force-frequency coefficient of singly rotated vibrating quartz crystals", IBM., J, Res., Dev., 12(1), pp. 92-99 (1968).

10. Dauwalter, C.R. "The temperature dependence of the force sensitivity of AT-cut quartz crystals", Proc. 26th. Annu. Freq. Contr. Symp., pp. 108-112 (1972).

11. Lee, P.C.Y., Wang, Y.S. and Markenscoff, X. "Highfrequency vibrations of crystal plates under initial stresses", J. Acoust. Soc. Am., 57(1), pp. 95-105 (1975).

12. Yang, J.S. and Zhang, X. "Vibration of a crystal plate under a thermal bias", J. Term. Stress., 26, pp. 467477 (2011).

13. Yong, Y., Patel, M.S. and Tanaka, M. "Frequencytemperature behavior of quartz resonators affected by transient and steady state temperature changes", Proc. Ultrason. Symp., Vancouver, BC., pp. 568-571 (2006).

14. Clayton, L.D. and EerNisse, E.P. "Frequency shift calculations for quartz resonators", in Proc. 45th. Annu. Freq. Contr. Symp., pp. 309-320 (1991).

15. Lee, P.C.Y. and Yong, Y.K. "Frequency temperature behavior of thickness vibrations of doubly rotated quartz plates affected by plate dimensions and orientations", J. Appl. Phys., 60(7), pp. 2327-2342 (1986).

16. Chen, J., Yong, Y.K., Kubena, R., Kirby, D. and Chang, D. "Application of electrode stress for improving frequency-temperature behavior of UHF quartz resonators", IEEE Int. Ultrason. Symp., pp. 1-4 (2015).

17. Chen, J., Yong, Y.K., Kubena, R., Kirby, D. and Chang, D. "Reduction of frequency deviations in quartz resonators by electric potentials of plate electrodes", IEEE Int. Ultrason. Symp., pp. 252-255 (2014).

18. Patel, M.S. "Nonlinear behavior in quartz resonators and its stability", Ph.D. dissertation, Dept. Civil Eng., New Brunswick., New Jersey Univ. (2008).

19. Wang, Z. Zhu, H. Dong, Y. Wang, J. and Feng, G. "Force-frequency coefficient of symmetrical incomplete circular quartz crystal resonator", IEEE Trans. Ultrason., Ferroelectr., Freq. Control., 48(5), pp. 1471-1479 (2001). 
20. Wang, J., Chen, Y., Wu, R., Wang, L., Jing, H., Du, J., Hu, Y. and Li, G., An Analysis of Nonlinear Thickness-shear Vibrations of Quartz Crystal Plates by Two-Dimensional Finite Element Method, arXiv preprint arXiv:1402.4753 (2013).

21. Beerwinkle, A.D., Nonlinear Finite Element Modeling of Quartz Crystal Resonators, M.S. Thesis, Dept. Mech. Eng., Oklahoma State Univ, Stillwater (2011).

22. Tiersten, H.F., Linear Piezoelectric Plate Vibrations: Elements of the Linear Theory of Piezoelectricity and the Vibrations Piezoelectric Plates, Springer (2013).

23. Mindlin, R.D. "First-order approximation", In An Introduction to the Mathematical Theory of Vibrations of Elastic Plates, J. Yong, Ed., pp. 115-151, World Scientific (2006).

24. Mindlin, R.D. "High frequency vibration of piezoelectric crystal plates", Int. J. Solids Structures., 8(7), pp. 895-906 (1972).

25. Bechman, R., Ballato, A.D. and Lukaszekt. T.J. "Higher-order temperature coefficients of the elastic stiff nesses and compliances of alpha-quartz", Proc. IRE. (1962).

26. Lee, P.C.Y. and Yong. Y.K. "Temperature derivatives of elastic stiffness derived from the frequency temperature behavior of quartz plates", J. Appl. Phys., 56(5), pp. 1514-1520 (1984).

27. Thurston, R.N., Mc Skimin, H.J. and Andreatch, P. "Third order elastic coefficients of quartz", J. Appl. Phys., 37, pp. 267-275 (1966).

28. Zhang, H., Turner, J.A., Yang, J. and Kosinski, J.A. "Force-frequency effect of thickness mode langasite resonators", Ultrason., 50(4), pp. 479-490 (2010).

29. Ballato, A., EerNisse, E.P. and Lukaszek, T. "The force-frequency effect in doubly rotated quartz resonators", 31st Ann. Symp. Freq. Control., pp. 8-16 (1977).
30. EerNisse, E.P. "Temperature dependence of the forcefrequency effect for the AT, FC, SC and rotated Xcuts", Proc. 34th Annu. Freq. Control. Symp., pp. 426430 (1980).

31. Mohammadi, M.M., Daneshpajooh, H. and Hamedi, M. "Effect of anisotropy and piezoelectricity on the force-frequency coefficient of AT-cut quartz crystals", Scientia Iranica B, 23(5), pp. 2203-2210 (2016).

\section{Biographies}

Mohammad Mostafa Mohammadi is a PhD graduate from School of Mechanical Engineering, University of Tehran. He obtained his MSc degree in Manufacturing Engineering from Tarbiat Modares University of Tehran, in 2007, and BS degree from Tabriz University in 2004. His $\mathrm{PhD}$ thesis is on the mathematical modelling of quartz resonator pressure sensors.

Mohsen Hamedi is a Professor of Manufacturing in University of Tehran where he teaches in graduate and undergraduate levels. His research interests are microfabrication, microsystems, and optimization of manufacturing processes. He has published more than 130 papers in international journals and conferences along with supervising more than $50 \mathrm{PhD}$ and master theses. He obtained his BSc and MSc degrees from University of Tehran and PhD from university of New Brunswick, Canada. He is a Founding Member of Iranian Society of Value Engineering, The R\&D Society of Iranian Industry, Iranian Society of Manufacturing Engineers and a member of Iranian Society of Mechanical Engineers.

Hossein Daneshpajooh is a $\mathrm{PhD}$ candidate in Department of Electrical Engineering, Pennsylvania State University, USA. He obtained his BSc degree from Amirkabir University of Technology, Tehran, Iran, and his MSc degree from University of Tehran, Iran. 